\title{
A biopsychological review of gambling disorder
}

This article was published in the following Dove Press journal:

Neuropsychiatric Disease and Treatment

23 December 2016

Number of times this article has been viewed

\section{Gabriel C Quintero}

Florida State University - Republic of Panama, Panama City, Panama
Correspondence: Gabriel C Quintero Florida State University - Republic of Panama, 227 City of Knowledge, Clayton, Panama City, Panama Tel +507 3170367

Fax +5073I70366

Email gquintero@fsu.edu
Abstract: The present review is an overview of previous experimental work on biopsychological aspects of gambling disorder. It includes the topics 1) gambling disorder from the neuroimaging and electroencephalography (EEG) perspective, 2) cognitive, executive functioning, and neuropsychological aspects of gambling disorder, and 3) rodent models of gambling disorder. Penalties and losses in gambling can differ in terms of brain activity. Also, specific patterns of brain activity, brain anatomical traits, EEG responses, and cognitive and executive performance can discriminate pathological gamblers from nonpathological gamblers. Also, pathological gamblers can display dysfunction in such brain areas as the insula, frontal lobe, and orbitofrontal cortex. Pathological gambling is a heterogeneous disorder that can vary depending on the severity of cognition, the style of gambling (strategic or not), the prospect of recovery, proneness to relapse, and proneness to treatment withdrawal. Finally, based on rodent models of gambling, the appropriateness of gambling decision is influenced by the presence of cues, the activity of dopamine receptors, and the activity of some brain areas (infralimbic, prelimbic, or rostral agranular insular cortex). Pathological gamblers differed in terms of frontoparietal brain activation compared to nonpathological gamblers (if winning or losing a game). Pathological gamblers had dysfunctional EEG activity. The severity of gambling was linked to the magnification and content of cognitive distortions. The insula was fundamental in the distortion of cognitions linked to result analysis during gambling activity.

Keywords: pathological gambling, biopsychology, human, rodent

\section{Introduction}

Gambling behavior can be defined as to put in risk something valuable, and relying on the expectation of obtaining a benefit in profit. ${ }^{1}$ Gaming disorder is distinguished by gaming behaviors that seriously alter the finances, social relationships, and the occupational progress of a subject. ${ }^{2}$ Gambling disorder has a life prevalence of $0.4 \%-4.2 \%{ }^{3}$ On the other hand, gambling disorder is currently classified in the Diagnostic and Statistical Manual of Mental Disorders (DSM)-5 in a new category, in the addictions section (behavioral addictions). ${ }^{4}$ However, it is important to note that some publications cited here will classify pathological gambling as an impulse disorder (rather than behavioral addiction), because that was the former classification (before 2013).

The ethnic group is an important variable that could influence the development (eg, prognosis, diagnosis) of a gambling disorder. Also, ethnic groups differ in their profile of psychiatric disorders. 5,6 Therefore, two subjects with gambling disorder that belong to different ethnic groups could display differences in gambling severity because of differences in psychiatric comorbidities. As an illustration, different researchers have evaluated relationships between gambling disorder and psychiatric disorders across different ethnic groups. ${ }^{5,6}$ Specifically, Barry et al ${ }^{5}$ studied a sample of 31,830 adult subjects ( $87 \%$ white and $13 \%$ Hispanic), and concluded that different degrees of gambling-disorder seriousness were related to the comorbidities 
of mental ailments (axes I and II) in whites and Latinos. Moreover, it was found that Hispanic subjects were the most probable to denote a gambling-related disorder (compared to white subjects). Furthermore, this study found a solid correlation amid moderate gambling problems and a wide variety of disorders of axis I (humor, eagerness, and drugconsumption ailments) and axis II (particularly group B) in Latino subjects in contrast to the white group. ${ }^{5}$ Another investigation studied a sample $(n=32,316)$ consisting of African-American and white adults to examine discrepancies in the relationships between gambling severity and mental ailments. ${ }^{6}$ This investigation affirmed that black subjects had a higher likelihood than white subjects to denote gambling problems, and a firmer relationship between gambling problems and humor disorder, low severity mania, and drug-consumption problems. ${ }^{6}$ In general, both studies stressed the relevance of considering race-linked variables in psychic health safeguards and therapeutic approaches for gambling problems. ${ }^{5,6}$

\section{Overview of experimental work on gambling disorder Neuroimaging and electroencephalography (EEG) perspective}

Specific patterns of brain-network activities are linked to penalties (losses) or recompenses (profits) of the gambling activity. ${ }^{7}$ It is even possible to differentiate between pathological gamblers and casual gamblers in terms of brain activation, ${ }^{8}$ amount of brain gray matter, ${ }^{9}$ size of specific brain structures, ${ }^{9}$ incidence of previous cerebral damage, and abnormal EEG responses. ${ }^{10}$

An illustration that it is possible to differentiate between pathological gamblers and casual gamblers in terms of brain activation is a study performed by Miedl et al. This contrasted a group of casual gamblers and problem gamblers during a simulation of a blackjack game by means of functional magnetic resonance imaging (fMRI). ${ }^{8}$ Specifically, levels of brain activity were measured during hazard evaluation (higher and lower hazard) and prize processing (win or lose money) by means of tasks that required the subjects to choose between taking or not a card in blackjack circumstances of varied levels of risk. No differences were found between groups in behavioral aspects; however, the indices linked to blood oxygen level differed markedly between groups in of the thalamus, superior temporal, and inferior frontal brain regions. While problem gamblers showed an augmented response under high-hazard circumstances and a reduction in low-hazard circumstances, occasional gamblers displayed the opposite response. Moreover, during recompense processing, both problematic and occasional gamblers showed an increase in brain activity in the posterior cingulated and ventral striatum. In addition, problematic players displayed a different activation pattern in the frontoparietal brain, which could represent a cue-elicited addiction memory matrix that was provoked by gaming-associated cues. ${ }^{8}$

Another investigation that illustrates that specific patterns of activity in brain networks are linked to penalties (losses) or recompenses (profits) of the gambling activity was performed by Camara et al. This work studied the processing of neural devices associated with the processing of penalties and recompenses. ${ }^{7}$ In particular, varied patterns of functional linkage (local brain areas and broader) were analyzed by means of fMRI while subjects executed a gaming exercise. The investigation found that monetary profits and losses activated similar brain areas (composed of frontal brain - striatum and limbic system); besides, the principal activation was detected in the lower striatum (in both hemispheres). Functional linkage assays displayed analogous reactions to gain and loss circumstances in the amygdala, hippocampus, and insular cortex that interrelated with the activation detected in the seedy area of the inferior striatum, and the linkage of the amygdala appeared more marked subsequently to losses. ${ }^{7}$

On the other hand, it is also possible to differentiate between pathological gamblers versus casual gamblers based on the amount of brain gray matter and the size of specific brain structures, based on research performed by Fuentes et al. ${ }^{9}$ This study evaluated brain-volume differences between pathological gamblers subjects with a gambling disorder $(n=30)$ and healthy volunteers $(n=30)$ by means of the analysis of images obtained from a structural magnetic resonance equipment $(1.5 \mathrm{~T}) .{ }^{9}$ The only difference found was augmented gray-matter volume in gamblers compared to the controls; also, gamblers displayed diminished sizes in the thalamus (right), hippocampus (right), and putamen (left). The main conclusion was that anatomical irregularities of the brain might foster activity changes linked to the signs of gambling disorder; also, this study supports the idea that the cerebrum's recompense system is important to the pathophysiology of this disease. ${ }^{9}$

Potenza et al contrasted a group of male subjects with gambling disorder and a control group by means of the use of images of event-related fMRI. ${ }^{11}$ Specifically, the activity of the prefrontal cortex (mainly the ventromedial area) of the subjects during Stroop-test performance was analyzed. Pathological gamblers displayed lower responses in the left 
ventromedial prefrontal cortex than control subjects when tested with infrequent incongruent stimuli. Nevertheless, both clusters displayed equivalent performance changes in different cerebrum areas, encompassing the activation of the superior rostral cingulate cortex and the dorsolateral frontal cortex. This study concluded that pathological gamblers and controls shared numerous neuronal interrelates during Stroop-test execution, but varied in a cerebrum zone related to impulsiveness. ${ }^{11}$

An illustration that it is also feasible to differentiate between pathological gamblers and casual gamblers based on the presence of previous cerebral damage and abnormal EEG profile was an investigation by Regard et al. This study contrasted a group of gamblers without substanceuse disorder and a group of healthy subjects, by means of a behavioral neurologic interview, neuropsychological evaluation, and EEG measurements. ${ }^{10}$ The study found that $81 \%$ of gamers had a health background of cerebral damage, and it was found that gamers were significantly more disrupted in memory, executive functions, and concentration. Also, EEG analysis showed impaired response in $65 \%$ of the players compared to $26 \%$ of controls. The investigation concluded that gamblers were cerebrum-impaired and had higher impairment in neuropsychological functions that relates to frontotemporolimbic circuits and more EEG-related irregularities. The investigators hypothesized that gambling disorder might be the result of cerebrum impairment, mainly in the frontolimbic system. ${ }^{10}$

Finally, another work by Doñamayor et al contrasted monetary gains and losses in gambling situations based on whole-head magnetoencephalography analysis. ${ }^{12}$ Specifically, losses were linked to mediofrontal feedback-related negativity and fluctuating response to a constraint in $\theta$-band frequency; nevertheless, gains were related to a burst in the $\beta$-range, based on endogenous-related potentials. Moreover, parallel analysis with whole-head magnetoencephalography found in the condition of losses that magnetic correlates of feedback-related negativity were extended between 230 and $465 \mathrm{~ms}$. Also, this was linked to a primary generator in the caudal cingulate cortex, followed by the rostral cingulate cortex and right insula; this impact was responsive to the extent of the financial loss. Finally, differences were also found between win and lose conditions in terms of oscillatory components displayed by whole-head magnetoencephalography: the "win" condition was associated with fluctuating elements in the $\alpha-, \theta$-, and high $\beta$-low $\gamma$ ranges, but loss condition was linked to a high $\beta$-range (linked to the size of the loss).

\section{Conclusions on neuroimaging and EEG perspective}

As an integration of the studies outlined herein thus far, the main ideas following in this section can be proposed. The main difference in brain activity between problem gamblers and occasional gamblers if winning or losing a game was that problem gamblers displayed a different frontoparietal activation pattern (this might be interpreted as a signal-triggered addiction memory matrix induced by playing linked signals). ${ }^{8}$ Nevertheless, both groups showed a raised response in the caudal cingulate cortex and the inferior striatum. ${ }^{8}$

Other studies have explored differences in brain activity of normal subjects during winning or losing a game. In general, winning or losing induced similar response patterns in the frontostriatolimbic matrix (main peaks in ventral striatum), amygdala, insular cortex, and hippocampus based on endogenous-related potentials; however, during losses, the connectivity of the amygdala appeared more pronounced. ${ }^{7}$ Also, losses were linked to mediofrontal feedback-related negativity and fluctuating responses with a burst in $\theta$-interval; nevertheless, gains were related to a burst in the $\beta$-range, based on endogenous-related potentials. ${ }^{12}$

Furthermore, for losses, based on whole-head magnetoencephalography, the magnetic correlate of feedback-related negativity extended between 230 and $465 \mathrm{~ms}$. Moreover, it was linked to a primary inducer in the caudal cingulate cortex, followed by the rostral cingulate cortex and the right insula; this consequence was responsive to the size of the economic loss. ${ }^{12}$ Besides, winning and losing differed in the oscillatory components displayed by the whole-head magnetoencephalography. Specifically, winnings were associated with fluctuating elements in the $\alpha-, \theta-$, and high $\beta$-low $\gamma$ ranges, but losses were linked to the high- $\beta$ range (linked with the size of the loss). ${ }^{12}$

The main differences in brain activity, based on fMRI, between problem and occasional gamblers under high- versus low-risk conditions were as follows. ${ }^{8}$ During high-risk situations, problem gamblers showed a higher response in the thalamus and inferior rostral and superior temporal zones compared to occasional gamblers. On the other hand, during low-risk situations, problem gamblers showed a lower response in the thalamus and inferior rostral and superior temporal zones compared to occasional gamblers.

There were anatomical discrepancies between gamblers and nongamblers. Gamblers displayed more gray-matter volume compared to healthy volunteers, according to structural MRI technology. ${ }^{9}$ Moreover, healthy volunteers had larger volumes of the right hippocampus, right thalamus, 
and left putamen compared to gamblers. ${ }^{9}$ Gamblers showed a lower level of activity in the brain region related to impulse control (ventromedial prefrontal cortex) compared to controls, based on event-related fMRI; ${ }^{11}$ however, there were no discrepancies in the responses of the rostral cingulate cortex or dorsolateral frontal cortex. ${ }^{11}$ Gamblers had dysfunctional EEG activity compared to healthy controls.

\section{Cognitive functioning, executive functioning, and neuropsychological aspects of gambling disorder}

Pathological gamblers can display dysfunctions in cognitive or executive processes, and these alterations differentiate them from nonpathological gamblers. Some of the neuropsychological dysfunctions found in pathological gamblers are marked impulsivity, ${ }^{13-17}$ cognitive rigidity, ${ }^{13,14,18}$ deficit in reaction suppression, ${ }^{14}$ imprecise response, ${ }^{19}$ disruption of inhibition process, ${ }^{13}$ slower time evaluation, ${ }^{13,14}$ disruption organizing tasks, ${ }^{13,20}$ impaired decisions $\left(\right.$ risky $^{21}$ or choosing), ${ }^{17}$ deficits assessing future results, ${ }^{22}$ memory impairments, ${ }^{10}$ concentration impairments, ${ }^{10}$ impaired executive performance, ${ }^{10}$ marked novelty searching, ${ }^{17}$ marked harm prevention, ${ }^{17}$ lack of cooperation, ${ }^{17}$ poor self-directedness, ${ }^{17}$ deficits in problem-solving (finding new procedures), ${ }^{23}$ and poor efficacy. ${ }^{23}$

Moreover, different neuropsychological alterations found in pathological gamblers have been linked to brain dysfunction in such areas as the insula (interpretation of events and results), ${ }^{24}$ frontal lobe (decreased executive performance), ${ }^{13}$ orbitofrontal cortex (impaired decisions, ${ }^{21}$ assessment of future results, ${ }^{22}$ or cognitive rigidity), ${ }^{18}$ prefrontal cortex (cognitive rigidity (ventrolateral zone), ${ }^{18}$ impaired decisions (dorsolateral zone)), ${ }^{21}$ deficits finding alternative procedures in problems, ${ }^{23}$ lower efficacy, ${ }^{23}$ and the ventral striatum (cognitive rigidity). ${ }^{23}$ Moreover, other pathological gamblers' dysfunctions involve such brain networks as the frontotemporolimbic matrix (deficits in memory, concentration, and executive performances) $)^{10}$ and frontotemporal area (deficits in choosing decision, impulsivity, upper novelty searching, higher harm prevention, reduced sense of cooperation, and decreased self-directedness). ${ }^{17}$

Even within clusters of subjects with pathological gambling, it is possible to find internal differences based on: the degree of cognitive distortion (marked distortions are linked to a more severe disorder); ${ }^{25}$ the style of gambling games (strategic vs non strategic games; pathological gamblers with diverse gambling styles can differ in terms of sex, marital status, and age); ${ }^{26}$ gambling-relapse proneness (some variables can influence gambling relapse, like duration of disorder, disinhibition, resolution selection, and internal neurocognitive features); ${ }^{13}$ proneness to treatment withdrawal (factors like high explorative excitability, self-regulatory disruptions, executive impairments, and high impulsivity could ease treatment withdrawal); ${ }^{27}$ and differences in recovery and treatment progress (substance-use disorders impair decision and control [inhibition] processes, because substances impair prefrontal cortex functioning). ${ }^{28}$ It has been reported that pathological gamblers can present parallel substance-use disorder; this combination of disorders can make recovery and/or treatment processes more difficult. ${ }^{29}$

Now, different studies are described that compose this section on cognition, executive function, and the neuropsychology of pathological gambling. First, a study explored the association between gambling-associated cognitive distortions and different degrees of gambling pathology (probable pathological play, probable problematic play, and unproblematic play). ${ }^{25}$ It employed youths, young adults, and mature adults from Chinese populations. Outcomes indicated that cognitive distortions, mainly those related to a perceived incapacity to stop gaming and favorable gaming anticipation, were salient signals of abnormal play in the three developmental groups. More specifically, it was reported that the presumable pathological gambling cluster had more cognitive distortions than the presumable problematic gambling cluster, which subsequently denoted more cognitive distortions than the unproblematic gambling cluster. Nevertheless, the degree of cognitive biases showed a different age tendency depending of the level of gambling problem: in the unproblematic gambling cluster, mature subjects displayed more cognitive distortions than the other clusters; on the other hand, in the probable problematic gambling cluster, mature subjects showed less cognitive distortions compared to other groups; and in the probable pathological gambling cluster, the youths displayed more cognitive distortions than the other clusters. Finally, sex differences were also reported in the cognitive biases: in the unproblematic and probable problematic gambling clusters, males showed an upper distortion in their recognized incapacity to end play as contrasted to females; on the other hand, in the probable pathological gambling cluster insignificant sex contrasts were reported. ${ }^{25}$

An investigation by Ledgerwood et al contrasted intellectual capacity, memory, and the executive functions (memory [working], reaction inhibition, cognitive plasticity, perseveration, decision elaboration, and organization) between groups of subjects with a gambling disorder and controls (sample 
of 45 subjects per cluster). ${ }^{20}$ The investigation reported that subjects with a gambling disorder displayed particular shortcomings in measurements of organization and decision elaboration compared to control subjects. ${ }^{20}$

Another work contrasted two groups of subjects with a gambling disorder $(\mathrm{n}=77)$ divided by the preferred forms of gambling: strategic versus nonstrategic. ${ }^{26}$ The strategic form of gambling consisted of craps, cards, competitive games, and the stock exchange; the nonstrategic gambling consisted of pull tabs, slot machines, and video poker. The clusters were compared based on different variables like clinical features (gambling severity, time, and money used), concurrent mental illness, and brain and cognition exams (cognitive plasticity and motor impetuosity). The study found that nonstrategic players were more probable to be women, divorced, and older; moreover, the amount of money used for play did not vary between clusters. Nonstrategic and strategic players did not vary in terms of cognitive performance: both clusters displayed dysfunction in inhibitory control and cognitive rigidity compared to control subjects. It was concluded that favorite ways of gaming (nonstrategic vs strategic) might be linked with particular clinical features but are not separable in terms of motor impetuosity and cognitive rigidity. ${ }^{26}$

Billieux et $\mathrm{al}^{30}$ evaluated if features linked to gaming cognition (eg, convictions that rituals might help to succeed in playing) could impact behavior and personal replies during experimental gambling. For this purpose, a group of subjects ( $\mathrm{n}=84)$ who played at least every month executed a noncomplex slot-machine exercise. The research found that ability-oriented gaming cognitions (eg, false imagination of influence promoted by subjective variables like reevaluation of negative outcomes), but not ritual-oriented gambling cognitions (eg, false imagination of influence promoted by outer variables like fortune), promoted higher personal scores in motivation to play following near-miss results. On the other hand, it was reported that an absence of personal control forecast perseverance on the slot-machine exercise. The research concluded that the stimulatory influence of near-miss results was associated with gambling cognitions related to ability acquisition, backing the notion that gambling near misses promote the appearance of control. ${ }^{30}$

An investigation evaluated a cluster of subjects aged 18-65 years; these subjects gambled and were recruited by means of newspaper advertising. ${ }^{14}$ Participants were clustered into three parties (subject without risk, subjects at risk, and subjects with gambling disorder) based on a diagnostic interview. The work found that subjects with a gambling disorder were remarkably aged and showed meaningful deficits related to movement impulsivity, response speed, and cognitive flexibility compared to control subjects. This work concluded that disabled reaction suppression and cognitive plasticity existed in subjects with a gambling disorder, contrasting with players without risk and at risk. Also, it concluded that the prompt recognition of this disorder in teenage or early adulthood stages may help in the prevention of gambling disorder starting. ${ }^{14}$

Kertzman et al contrasted interference control in a cluster of fellows with a gaming disorder $(\mathrm{n}=62)$ and control subjects $(n=83)$ by means of the reverse alternative of the Stroop task. ${ }^{19}$ It was found that the performance of subjects with gambling disorder was significantly imprecise and slower compared to controls. In addition, the mean response time in the neutral state (words in black ink) was slow compared to the mean response time in the incongruent state (color name and ink different). This work concluded that the execution of the Stroop test was disrupted in the pathological gamblers. ${ }^{19}$

Goudriaan et al evaluated neurocognitive disabilities of executive functions in groups of subjects with gambling disorder $(n=49)$, normal controls $(n=49)$, those with substance-use disorder (alcohol-use disorder, $\mathrm{n}=46$ ), and a cluster with disturbance in control of impulses (Tourette's, $\mathrm{n}=46) \cdot{ }^{13} \mathrm{~A}$ broad neuropsychological battery was employed that measured executive performance and basic cognitive performance. It was discovered that groups of participants with gambling or alcohol-use disorders displayed shortages on inhibition, time evaluation, cognitive plasticity, and organizing tasks. The main conclusion of this study was that subjects with gambling and alcohol-use disorders were distinguished by decreased executive performance; this suggests an underlying impairment in frontal lobe connectivity. The similarity between the gambling and alcohol-use disorder clusters suggested a common neurocognitive etiology for these diseases. ${ }^{13}$

Another study contrasted a group of male subjects with a gambling disorder $(n=25)$ and a group of male controls $(n=25)$ by means of the game-of-dice task. ${ }^{21}$ This investigation found that subjects with a gambling disorder exhibited marked deficits in the game-of-dice task; moreover, it was discovered that the incidence of hazardous resolutions was related to feedback analysis and executive performances. The study concluded that risky decisions by subjects with a gambling disorder might be impacted by orbitofrontal and dorsolateral prefrontal impairments. ${ }^{21}$

On the other hand, Cavedini et $\mathrm{al}^{22}$ contrasted the decision-elaboration processes executed by the activity of 
the inferomedial prefrontal cortex in a group of pathological gamblers $(n=20)$ and healthy control subjects $(n=40)$ by means of a gambling task. The study suggested the presence of a relationship among gambling disorder and diverse diseases (eg, substance-use disorder and obsessivecompulsive disorder) that displayed a reduced capacity to assess future results, and moreover that this could be accounted for at least partially by atypical performance of the orbitofrontal cortex. ${ }^{22}$

Boog et al studied cognitive rigidity in a group of pathological gamblers by means of tasks: the first implicating cognitive rigidity with a recompense component (eg, reversed apprenticeship), and the second an exercise evaluating overall cognitive rigidity out of such an element (response perseverance). ${ }^{18}$ For this aim, the ratings of a recompense-based reversed-apprenticeship exercise (probabilistic reversed-apprenticeship exercise) and the Wisconsin Card Sorting Test (WCST) were contrasted among a cluster of therapy-seeking subjects with gambling disorder and a control cluster (matched by age and sex). The outcomes demonstrated that subjects with gambling disorder had disrupted execution only on the neurocognitive exercise evaluating recompense-based cognitive rigidity. The findings suggested that cognitive inflexibility in subjects with gambling disorder is the consequence of an abnormal recompense-based apprenticeship, and not grounded on broader trouble with cognitive rigidity. Moreover, the researchers concluded that the pattern of troubles noted was a signal of dysfunction of the ventrolateral prefrontal cortex, orbitofrontal cortex, and the ventral region of the striatum in subjects with a gambling disorder. ${ }^{18}$

Marazziti et al explored the pathophysiology of gambling disorder. ${ }^{23}$ The study analyzed a group of subjects with a gambling disorder $(n=20)$ using neuropsychological exams for the purpose of exploring the cerebrum zones linked to the disease. The tests employed were the verbal associative fluency test, the WCST, and the Wechsler Memory Scale (revised). Compared to the control group, the subjects with a gambling disorder displayed differences only in the WCST; specifically, they showed more deficits in discovering optional procedures for problem-solving and displayed a decrement in efficacy as they advanced across the successive stages of the task. The average ratings of the other trials were inside the standard range. The study concluded that subjects with a gambling disorder had deficits stemming from the WCST; specifically, they were unable to learn from their errors and to search for other answers. It also concluded that abnormal activity in the prefrontal zones might induce in pathological gamblers a sort of cognitive rigidity that might make them susceptible to the evolution of impetuous and/or compulsive conducts, like those found in gambling disorder.

On the other hand, using fMRI, Coricelli et al reported that recovery of response in the amygdala and orbitofrontal cortex happened during the stage of selection, when the cerebrum was expecting potentially successful outcomes of resolutions. ${ }^{31}$ Furthermore, these patterns mirrored apprenticeship based on previously collected emotional situations. Also, emotional outcomes were able to generate determined processes of cognitive monitoring during the selection processes, implicating strengthening or prevention of the encountered conduct.

Bechara and Martin explored if substance dependence could impair working memory based on the gaming exercise and the retarded non-matching to sample exercise. ${ }^{28}$ Based on their results, the authors proposed that the prefrontal cortex was in control of diverse mechanisms of decision making and inhibitory control. Also, they proposed that subjects with substance-use disorder were affected in any one or combination of them. These results were important, because it is common to find pathological gamblers displaying parallel substance-use disorders, and this condition can influence therapeutic and recovery processes. ${ }^{29}$

On the other hand, Goudriaan et $\mathrm{al}^{32}$ sought elucidation of factors that influence relapse in gambling disorder. For this purpose, they used a sample of subjects with a gambling disorder $(n=46)$, and investigated the effects of impulsivity, reward sensitivity, disinhibition, and selection processes (under contradictory circumstances) on gambling-disorder relapse. The work found that prolonged length of the disease, neurocognitive markers of disinhibition (the reaction time for stop signal), and selection of the resolution process (card-playing test) were meaningful forecasters of relapse (accounting for roughly 53\% of the variance). Conversely, recompense sensitivity and impetuosity did not forecast gambling-disorder relapse. The investigation concluded that the duration of the disorder, disinhibition measurements, and resolution selection were strong forecasters of relapse. Moreover, the findings pointed to internal neurocognitive features being more reliable in the forecasting of relapse compared to external personality features. ${ }^{32}$

Regard et al contrasted a group of players without substance-use disorder $(\mathrm{n}=21)$ and a group of healthy subjects $(n=19)$ by means of a behavioral neurologic interview (centered on potential cerebrum impairment), EEG, and neuropsychological assessment. The study found that $81 \%$ of gamblers had a positive health background for cerebral 
impairment; also, gamblers were more disrupted compared to controls in memory, concentration, and executive performance. Also, EEG showed an abnormal response in $65 \%$ of the players, contrasted to $26 \%$ of controls. The investigation concluded that players were cerebrum damaged and had augmented neuropsychological abnormalities of the frontotemporolimbic brain matrix and augmented EEG-related anomalies. The investigators hypothesized that gambling disorder was the result of cerebrum impairment, specifically of the frontolimbic circuits. ${ }^{10}$

Another recent study compared sufferers with different precise injuries in the brain (amygdala, insula, or inferomedial prefrontal cortex), healthy control subjects, and subjects with diverse brain lesions. ${ }^{24}$ As a part of the study, the participants were required to perform games in the roulette and slotmachine devices. It was concluded that the altered cognitive handling of near misses and event succession was normally realized by means of insula activity. Moreover, the study concluded that a therapeutic approach that reduces insula reactivity could be useful for treating gambling disorders. ${ }^{24}$

Other research in Chinese men contrasted pathological gamblers $(n=37)$ and controls $(n=40)$ for elucidating the relationship between gambling disorder and impulsivity. ${ }^{15}$ The research found that those with a gaming disorder were remarkably more impulsive compared to controls. Nevertheless, no discrepancies were found between the clusters on the emotional conflict test or the Stroop color-word test. It was concluded that gambling disorder was linked to trait impetuosity, rather than state impetuosity. More specifically, gambling disorder was linked to a type of impetuosity originating from long-lasting personality features that direct gamers to center on near-term profits (trait impetuosity), rather than temporary cognitive or emotional disinhibition (state impetuosity). Furthermore, the study recommended that therapy focus on changing gamblers' regular execution by promoting salutary reflection practices and centering on delayed-term recompense. ${ }^{15}$

Alvarez-Moya et $\mathrm{al}^{27}$ explored relationships among selfinformed impetuosity, neurocognitive indices, and therapy results in gambling disorder. This investigation employed a cluster of subjects with a gambling disease (sample of 88 subjects), but it lacked a control group. The subjects were evaluated by means of tests that measured executive functions, decision making, and impetuosity. The method of treatment employed was cognitive behavioral therapy. This investigation found that there was a high number of uncommon results (in participants self reports) which were linked to low performance in the Iowa Gambling test. Furthermore, elevated explorative excitability, elevated impetuosity, deficient reverse-block span, and poor Iowa Gambling Task (EFGH scores) forecast treatment withdrawal. Neither the self-informed index nor neurocognitive index was related to setbacks or number of therapy sessions. It was concluded that neurocognitive recompense sensitivity was associated with participants self reported behavior about excesses in expenditure. Self-regulatory disruptions (mainly penalty sensitivity and reckless impetuosity) and executive impairment forecast dropout from cognitive behavioral therapy in pathological gamblers. It was also concluded that distinct personality characteristics and neurocognitive processes modulated gamblers' reactions to mental treatment, depending on the particular variable evaluated. ${ }^{27}$

Fuentes et $\mathrm{al}^{16}$ compared 214 subjects with a gambling disorder $(24.3 \%$ without a parallel disorder and $75.7 \%$ with a parallel disorder) and 82 controls based on the response times, frequency of mistakes (go/no-go exercises), and Barratt Impulsiveness Scale ratings. The subjects with a gambling disorder made more mistakes at the go/no-go exercises and showed higher ratings on the Barratt Impulsiveness Scale. Moreover, the authors proposed that neuropsychology tests and the Barratt Impulsiveness Scale integrated a multinomial logistic design that differentiated subjects with a playing disorder from those without a playing disorder; furthermore, this design was better than other designs with a single sort of measurement. According to the results, impetuosity was an experience with multiple dimensions, and gamblers were a large and varied cluster with different degrees of impetuosity. ${ }^{16}$

Another study explored the forecasting of variance in personality and neuropsychological features in subjects with gambling disorder. ${ }^{17}$ Subjects with gambling disorder $(n=25)$ and a control group $(n=34)$ were contrasted by means of the Barratt Impulsiveness Scale, Temperament and Character Inventory, and neuropsychological exams. Those with a gambling disorder displayed frontotemporal impairment based on neuropsychological tests, and displayed deficits related to choice (Iowa Gambling Test), excess of impetuosity, higher novelty-searching, higher harm prevention, reduced sense of cooperation, and a decreased degree of self-directedness. Studies of logistical regression showed that neuropsychological factors did not augment significantly the variance over personality features in forecasting gambling disorder; however, personality factors enhanced meaningful incremental variance above neuropsychological features in forecasting gambling disorder. The main conclusion was that personality traits were more suitable forecasters of gambling disorder compared to neuropsychological characteristics. ${ }^{17}$ 


\section{Conclusions on cognitive functioning, executive functioning, and neuropsycho- logical aspects of gambling disorder}

The magnification and content of gambling-related cognitive biases are related to the severity of gambling problem. Specifically, the strength of cognitive biases was related to the seriousness of the gambling disease (eg, probable pathological playing group $>$ probable problem-playing group $>$ non-problem-playing group) according to a Chinese study. ${ }^{25}$ The youths were the age-group of pathological gamblers with higher cognitive biases (compared to young adults and mature adults), and there was no evidence of sex differences. ${ }^{25} \mathrm{On}$ the other hand, cognition influenced by gambling skills (but not cognition influenced by rituals) predicted desire for play following near-miss results; furthermore, a sensed lack of personal control predicted perseverance on the slot-machine test (based on artificial lab conditions). ${ }^{30}$

In terms of normal general functioning, human decisions are not only rational but also strongly influenced by emotions. ${ }^{31}$ More specifically, remorse (an emotion) guides choice behaviors, and remorse experience is mediated by activity of the orbitofrontal cortex. ${ }^{31}$

Studies that elucidated the relationship among brain activity, cognitive processes, and decision processes suggested the orbitofrontal cortex, amygdala, and insula as fundamental structures. Specifically, the activation of the amygdala and the orbitofrontal cortex occurred during the selection process: the cerebrum analyzed possible consequences of decisions and anticipation of regret. ${ }^{31}$ Moreover, the insula was fundamental in altered cognitive interpretation of near-miss results and trial sequences in gambling-related tasks. ${ }^{24}$

Different studies have supported neuropsychological differences between subjects with gambling disorder and control subjects. Specifically, subjects with gambling disorder were older, with more deficits in motor-impulse control, deficits in response speed, deficits in cognitive plasticity, ${ }^{14}$ problems of organization, deficits in selection of the decision process, ${ }^{20}$ poorer inhibition, less accurate temporal estimation, poorer results in planning tests, ${ }^{13}$ deficits in the game-of-dice task, ${ }^{21}$ reduced ability to assess future consequences ${ }^{22}$ were slower, less accurate, and had impaired performance on the reverse Stroop. ${ }^{19}$ Furthermore, those with gambling disorder (compared to control subjects) were also impaired on the neurocognitive test evaluating recompense-based cognitive rigidity, ${ }^{18}$ displayed deficits in discovering alternative ways of resolving problems (WCST test), had decreased efficiency (WCST test), were unable to learn from errors and search for alternative responses, ${ }^{23}$ were remarkably disrupted in executive processing, and impaired in focus attention and memory. ${ }^{10}$

Neuropsychological research on subjects with a gambling disorder suggested that these subjects could have dysfunction in the insula (altered cognitive interpretation of near-miss results and trial success), ${ }^{24}$ frontal lobe (diminished executive functioning), ${ }^{13}$ ventral striatum (impairment on rewardbased cognitive flexibility), ${ }^{18}$ frontotemporolimbic circuits (impairments in concentration, memory, and executive functions) ${ }^{28}$ prefrontal cortex (cognitive rigidity, impulsivity, and compulsivity) ${ }^{23}$ dorsolateral prefrontal cortex (risky decisions), ${ }^{21}$ ventrolateral prefrontal cortex (impairment in reward-based cognitive flexibility), ${ }^{18}$ and orbitofrontal cortex (risky decisions, ${ }^{21}$ disability assessing future consequences, ${ }^{22}$ and impairment in reward-based cognitive flexibility). ${ }^{18}$

Impulsivity was one of the core characteristics of gambling disorder; in effect, different studies described subjects with gambling disorder as displaying more impulsivity ${ }^{15,17}$ and higher impulsivity scores (Barratt Impulsivity Scale). ${ }^{16}$ Moreover, other studies described subjects with gambling disorder as displaying trait-type (rather than state-type) impulsivity, ${ }^{15}$ and making more mistakes on the go/no-go exercise. ${ }^{16}$

A cluster of variables predicted relapse and treatment withdrawal in subjects with gambling disorder. Specifically, some predictors of relapse were longer gambling-disease duration, neurocognitive markers of impairment on disinhibition and resolution selection, and endophenotypic neurocognitive characteristics. ${ }^{32}$ On the other hand, some predictors of treatment withdrawal were impulsiveness, high exploratory excitability, poor results in the backward block-span test, and poor results in the Iowa Gambling Test (EFGH scores). ${ }^{27}$ Moreover, personal regulatory disabilities (rash impetuosity and penalty sensibility) and executive impairment forecast dropping out of therapy (cognitive behavioral). ${ }^{27}$

For the treatment of gambling disorder, it is necessary to consider if the subject has a parallel substance-use disorder, because this could worsen the gambling disorder. In effect, subjects with substance-use disorders can be impaired in any of the multiple processes of decision choice and inhibitory monitoring sited in the prefrontal cortex. ${ }^{28}$ Therefore, the parallel coexistence of a gambling disorder and a substanceuse disorder makes the treatment more challenging.

\section{Overview of experimental work on rodent gambling models}

Rodent models have suggested that some conditions can ease unfavorable or risky decisions, like the presence of 
audiovisual cues, ${ }^{33}$ the agonism of dopamine receptors $\left(D_{3}\right.$ type $),{ }^{33}$ and decreased activity in brain areas like the infralimbic (IL) or prelimbic (PrL) cortex. ${ }^{34}$ On the other hand, other factors like inactivation of rostral agranular insular cortex (RAIC) favored the selection of optimal decisions. ${ }^{35}$ Now, we examine studies that support the previous argument.

Different investigations have explored the role of brain functioning in gambling by means of rodent gambling models. ${ }^{33-35}$ A study performed in male Long Evans rats explored the relevance of audiovisual cues in facilitating dysfunctional choices in gambling tasks. ${ }^{33}$ For this purpose, the rat gambling task (rGT; cued and uncued forms) was employed, which is analogous to the human Iowa Gambling Task. As a reference, in the rGT the rodents had to select among four alternative responses that differed in terms of frequency and strength of reward and punishment. The main finding was that adding audiovisual cues to the task increased the selection of unfavorable risky options (despite the reinforcement contingencies being similar). Moreover, it was found that $\mathrm{D}_{3}$-receptor agonism facilitated the selection of unfavorable alternatives on only the cued-task version. On the other hand, $\mathrm{D}_{3}$-receptor antagonism had the inverse effect. Barrus and Winstanley proposed that analogous nervous processes are relevant to the capacity of cues to influence animal selection (preference toward unfavorable options) and ease substance-use disorder. ${ }^{33}$

Another investigation evaluated the relevance of different cortical regions and $\mathrm{D}_{2}$-receptor activity in rat decisionmaking processes by means of the rGT. ${ }^{34}$ Specifically, the PrL, IL, orbitofrontal, and anterior cingulate cortices were evaluated. After training in the rGT, the male Long Evans rats received cortical infusions of a combination of baclofen and muscimol or $\mathrm{D}_{2}$-receptor antagonists. It was found that inactivation of the IL or PrL cortex favored preference for unfavorable options and discouraged preference for favorable options. On the other hand, inactivation of the orbitofrontal cortex or anterior cingulate cortex did not alter decision making. Finally, infusion of the $\mathrm{D}_{2}$-receptor antagonist had no effect on decision making. ${ }^{34}$

Finally, additional research by Pushparaj contrasted the effects of pharmacological inactivation or lesioning of the RAIC and the caudal granular insular cortex of male Long Evans rats in performance on the rGT. ${ }^{35}$ It was found that inactivation of the RAIC (by means of local infusions of $\gamma$-aminobutyric acid after rGT training or lesioning of the RAIC before rGT training) made rats choose alternatives with greater reward frequency and lower punishment.

\section{Conclusions about the experimental work on rodent gambling models}

Based on rGT models, it seems that the following conditions could favor the selection of unfavorable or risky decisions: addition of audiovisual cues, ${ }^{33} \mathrm{D}_{3}$-receptor agonism (only during the presence of audiovisual cues), ${ }^{33}$ and inactivation of the IL or PrL (non-D 2 -receptor-dependent) cortices. ${ }^{34}$ On the other hand, it seems that inactivation of the RAIC by means of local infusions of $\gamma$-aminobutyric acid or lesions of the RAIC could favor the selection of alternatives with lower punishments or risks. ${ }^{35}$ It seems that $\mathrm{D}_{2}$-receptor antagonists (at least in the PrL, IL, orbitofrontal, or anterior cingulate cortices) do not influence the decision-making process. ${ }^{34}$

\section{Acknowledgments}

This work was funded by the SNI (Sistema Nacional de Investigacion - National System of Investigation) contract 106-2015 (awarded to GCQ). The SNI is a department that belongs to the SENACYT (Secretaria Nacional de Ciencia, Tecnologia e Innovacion - National Secretariat of Science, Technology and Innovation). SENACYT is physically located in the Republic of Panama.

\section{Disclosure}

The author reports no conflicts of interest in this work.

\section{References}

1. Potenza MN, Kosten TR, Rounsaville BJ. Pathological gambling. JAMA. 2001;286(2):141-144.

2. National Opinion Research Center. Gambling impact and behavior study. 1999. Available from: http://www.norc.org/pdfs/publications/ gibsfinalreportapril1999.pdf. Accessed November 29, 2016.

3. Lorains FK, Cowlishaw S, Thomas SA. Prevalence of comorbid disorders in problem and pathological gambling: systematic review and metaanalysis of population surveys. Addiction. 2011;106(3):490-498.

4. American Psychiatric Association. Diagnostic and Statistical Manual of Mental Disorders. 5th ed. Arlington, VA: APA; 2013.

5. Barry DT, Stefanovics EA, Desai RA, Potenza MN. Gambling problem severity and psychiatric disorders among Hispanic and white adults: findings from a nationally representative sample. J Psychiatr Res. 2011;45(3): 404-411.

6. Barry DT, Stefanovics EA, Desai RA, Potenza MN. Differences in the associations between gambling problem severity and psychiatric disorders among black and white adults: findings from the National Epidemiologic Survey on Alcohol and Related Conditions. Am J Addict. 2011;20(1):69-77.

7. Camara E, Rodriguez-Fornells A, Münte TF. Functional connectivity of reward processing in the brain. Front Hum Neurosci. 2008;2:19.

8. Miedl SF, Fehr T, Meyer G, Herrmann M. Neurobiological correlates of problem gambling in a quasi-realistic blackjack scenario as revealed by fMRI. Psychiatry Res. 2010;181(3):165-173.

9. Fuentes D, Rzezak P, Pereira FR, et al. Mapping brain volumetric abnormalities in never-treated pathological gamblers. Psychiatry Res. 2015; 232(3):208-213

10. Regard M, Knoch D, Gütling E, Landis T. Brain damage and addictive behavior: a neuropsychological and electroencephalogram investigation with pathologic gamblers. Cogn Behav Neurol. 2003;16(1):47-53. 
11. Potenza MN, Leung HC, Blumberg HP, et al. An FMRI Stroop task study of ventromedial prefrontal cortical function in pathological gamblers. Am J Psychiatry. 2003;160(11):1990-1994.

12. Doñamayor N, Marco-Pallarés J, Heldmann M, Schoenfeld MA, Münte TF. Temporal dynamics of reward processing revealed by magnetoencephalography. Hum Brain Mapp. 2011;32(12):2228-2240.

13. Goudriaan AE, Oosterlaan J, de Beurs E, van den Brink W. Neurocognitive functions in pathological gambling: a comparison with alcohol dependence, Tourette syndrome and normal controls. Addiction. 2006;101(4):534-547.

14. Odlaug BL, Chamberlain SR, Kim SW, Schreiber LR, Grant JE. A neurocognitive comparison of cognitive flexibility and response inhibition in gamblers with varying degrees of clinical severity. Psychol Med. 2011; 41(10):2111-2119.

15. Lai FD, Ip AK, Lee TM. Impulsivity and pathological gambling: Is it a state or a trait problem? BMC Res Notes. 2011;4:492.

16. Fuentes D, Tavares H, Artes R, Gorenstein C. Self-reported and neuropsychological measures of impulsivity in pathological gambling. $J$ Int Neuropsychol Soc. 2006;12(6):907-912.

17. Forbush KT, Shaw M, Graeber MA, et al. Neuropsychological characteristics and personality traits in pathological gambling. CNS Spectr. 2008;13(4):306-315.

18. Boog M, Höppener P, van der Wetering BJ, Goudriaan AE, Boog MC, Franken IH. Cognitive inflexibility in gamblers is primarily present in reward-related decision making. Front Hum Neurosci. 2014;8:569.

19. Kertzman S, Lowengrub K, Aizer A, Nahum ZB, Kotler M, Dannon PN. Stroop performance in pathological gamblers. Psychiatry Res. 2006; 142(1):1-10.

20. Ledgerwood DM, Orr ES, Kaploun KA, et al. Executive function in pathological gamblers and healthy controls. J Gambl Stud. 2012;28(1): 89-103.

21. Brand M, Kalbe E, Labudda K, Fujiwara E, Kessler J, Markowitsch HJ. Decision-making impairments in patients with pathological gambling. Psychiatry Res. 2005;133(1):91-99.

22. Cavedini P, Riboldi G, Keller R, D’Annucci A, Bellodi L. Frontal lobe dysfunction in pathological gambling patients. Biol Psychiatry. 2002;51(4):334-341.

23. Marazziti D, Dell'Osso MC, Conversano C, et al. Executive function abnormalities in pathological gamblers. Clin Pract Epidemiol Ment Health. 2008;4:7.
24. Clark L, Studer B, Bruss J, Tranel D, Bechara A. Damage to insula abolishes cognitive distortions during simulated gambling. Proc Natl Acad Sci U S A. 2014;111(16):6098-6103.

25. Tang CS, Wu AM. Gambling-related cognitive biases and pathological gambling among youths, young adults, and mature adults in Chinese societies. J Gambl Stud. 2012;28(1):139-154.

26. Grant JE, Odlaug BL, Chamberlain SR, Schreiber LR. Neurocognitive dysfunction in strategic and non-strategic gamblers. Prog Neuropsychopharmacol Biol Psychiatry. 2012;38(2):336-340.

27. Alvarez-Moya EM, Ochoa C, Jimenez-Murcia S, et al. Effect of executive functioning, decision-making and self-reported impulsivity on the treatment outcome of pathologic gambling. J Psychiatry Neurosci. 2011; 36(3):165-175.

28. Bechara A, Martin EM. Impaired decision making related to working memory deficits in individuals with substance addictions. Neuropsychology. 2004;18(1):152-162.

29. Grant JE, Chamberlain SR. Gambling disorder and its relationship with substance use disorders: implications for nosological revisions and treatment. Am J Addict. 2015;24(2):126-131.

30. Billieux J, Van der Linden M, Khazaal Y, Zullino D, Clark L. Trait gambling cognitions predict near-miss experiences and persistence in laboratory slot machine gambling. Br J Psychol. 2012;103(3):412-427.

31. Coricelli G, Dolan RJ, Sirigu A. Brain, emotion and decision making: the paradigmatic example of regret. Trends Cogn Sci. 2007;11(6): 258-265.

32. Goudriaan AE, Oosterlaan J, De Beurs E, Van Den Brink W. The role of self-reported impulsivity and reward sensitivity versus neurocognitive measures of disinhibition and decision-making in the prediction of relapse in pathological gamblers. Psychol Med. 2008;38(1):41-50.

33. Barrus MM, Winstanley CA. Dopamine D3 receptors modulate the ability of win-paired cues to increase risky choice in a rat gambling task. J Neurosci. 2016;36(3):785-794.

34. Zeeb FD, Baarendse PJ, Vanderschuren LJ, Winstanley CA. Inactivation of the prelimbic or infralimbic cortex impairs decision-making in the rat gambling task. Psychopharmacology (Berl). 2015;232(24): 4481-4491.

35. Pushparaj A, Kim AS, Musiol M, et al. Differential Involvement of the agranular vs granular insular cortex in the acquisition and performance of choice behavior in a rodent gambling task. Neuropsychopharmacology. 2015;40(12):2832-2842.
Neuropsychiatric Disease and Treatment

\section{Publish your work in this journal}

Neuropsychiatric Disease and Treatment is an international, peerreviewed journal of clinical therapeutics and pharmacology focusing on concise rapid reporting of clinical or pre-clinical studies on a range of neuropsychiatric and neurological disorders. This journal is indexed on PubMed Central, the 'PsycINFO' database and CAS,

\section{Dovepress}

and is the official journal of The International Neuropsychiatric Association (INA). The manuscript management system is completely online and includes a very quick and fair peer-review system, which is all easy to use. Visit http://www.dovepress.com/testimonials.php to read real quotes from published authors. 